\title{
Amphetamine psychosis: a model for studying the onset and course of psychosis
}

\author{
Daniel F Hermens, Dan I Lubman, Philip B Ward, Sharon L Naismith and Ian B Hickie
}

$\mathrm{N}$ euroscience research within the psychiatric field has tended to focus on detection of "unique" genetic factors and related "endophenotypes" (ie, intermediate phenotypes, assumed to have simpler genetic underpinnings than the disorder itself) in an attempt to identify potential aetiological mechanisms. ${ }^{1}$ In contrast, limited research has examined exposure to known environmental pathogens as a method of elucidating underlying neurobiological processes. Researchers in the United Kingdom have detailed a "bootstrapping" approach that starts with a known environmental pathogen and links it to specific illness states and possible at-risk genotypes. ${ }^{1}$ The goal is to achieve convergence, such that the environmental and genetic evidence is directly linked to the same underlying neural substrate. ${ }^{1}$ They stress that knowing all three factors, rather than the two that dominate most linear models, enhances the likelihood of elucidating the actual underlying pathophysiology. ${ }^{1}$

Here, we explore the capacity for amphetamine-type stimulants (ATSs) to function as a candidate environmental pathogen linked to schizophrenia and related psychoses. The potential to unravel relevant genotypes or causal neural substrates in cohorts of people exposed to ATSs is evaluated. ATS exposure is particularly attractive to researchers, as it is a common environmental pathogen. Further, the neurochemistry of acute intoxication is well understood. Neurophysiological and neuropsychological markers are available for schizophrenia and related psychoses. Some of these appear to be viable endophenotypes in association studies. ${ }^{2,3}$ If one accepts the basic proposition that ATS-related psychoses are biological equivalents of schizophrenia and related psychoses, this is an experimental model worthy of closer neurobiological and longitudinal examination (Box 1).

\section{Amphetamine-type stimulant-related psychosis}

ATS use increases the likelihood of experiencing psychotic symptoms, ${ }^{4,5}$ and the clinical characteristics of ATS-related psychosis can be indistinguishable from acute paranoid schizophrenia (Box 2). ${ }^{4}$ In this article, we use the term "ATS-related psychosis" to refer to the presence or history of psychotic symptoms associated with ATS use (ie, symptoms that emerge concurrently with or after ATS use, and were not present before ATS use). The model proposed here is particularly relevant to studies of young people (typically, 15-30 years), as psychotic symptoms are more likely to emerge during this age range.

ATS-related psychosis is thought to be most evident with methamphetamine use, which is currently the most commonly used illicit ATS in Australia. Almost one in 10 Australians (9.1\%) have tried methamphetamine, sold on the street under a variety of names, including "speed" and "ice". ${ }^{6}$ Early-onset ATS use, typically occurring in the mid-to-late teens, predicts later problems and increases the chance of developing psychosis. ${ }^{4,7}$ ATS users are at particularly high risk of experiencing precipitated or exacerbated psychotic symptoms, with an estimated prevalence of psychosis that is 11 times higher than the general population. ${ }^{5}$ Compared with other substances, methamphetamine is highly neurotoxic, and

\section{ABSTRACT}

- The aetiology of schizophrenia remains complex, although proposed models have identified genetic markers and environmental pathogens as important risk factors.

- Researchers have found no large-effect or unique genetic elements, and only a small number of putative environmental agents have been identified.

- Use of amphetamine-type stimulants (ATSs) is an exemplar environmental pathogen, as it is known to trigger schizophrenia-like illness and other psychotic and manic episodes.

- To date, the ATS model of illness onset has been underutilised. It has the potential to reveal key neurobiological elements of schizophrenia and related psychoses.

- The model proposed here has the capacity to inform detection of those at risk of ATS-related psychoses, and therefore help develop early intervention strategies.

- It is possible that the same approach may be used in young people known to be at risk of schizophrenia and related disorders, by informing models that involve other environmental or genetic risks.

MJA 2009; 190: S22-S25

its use can lead to long-term damage, with concomitant deleterious effects on cognition. ${ }^{8}$ Once ATS-related psychosis has developed, recurrence of psychotic symptoms can develop without ATS use. ${ }^{4}$ However, current narrow diagnostic definitions in the Diagnostic and statistical manual of mental disorders, fourth edition suggest that some ATS users who continue to experience psychotic symptoms for prolonged periods (beyond 1 month) should be diagnosed with a formal psychotic disorder (eg, schizophreniform disorder or schizophrenia), ${ }^{9}$ despite limited evidence for the validity of such criteria. ${ }^{10}$ Diagnostic issues are further compounded by findings that young people presenting with their first episode of psychosis have high rates of concurrent substance-use disorders. ${ }^{11}$

As with exposure to any other environmental pathogen, not all ATS users develop psychosis. The development of a psychotic episode is assumed to be related to additional vulnerability factors. ${ }^{7}, 12$ Traditionally, ATS-related psychosis is seen as distinct from schizophrenia, with the latter being assumed to be a progressive or chronic deteriorating disorder. ${ }^{12}$ However, ATS-related psychosis may persist well beyond periods of acute ATS intoxication, and may recur despite abstinence. ${ }^{8}$ In clinical practice, the dominant model has been a poorly validated hierarchical approach, whereby a diagnosis of schizophrenia or another psychosis is deferred in the presence of prior or ongoing ATS use, unless the patient has been intoxicated with, or withdrawn from, the ATS for over 1 month. ${ }^{13}$

Attempts at phenotypic differentiation of ATS-related psychosis from schizophrenia and related psychoses have been of limited 


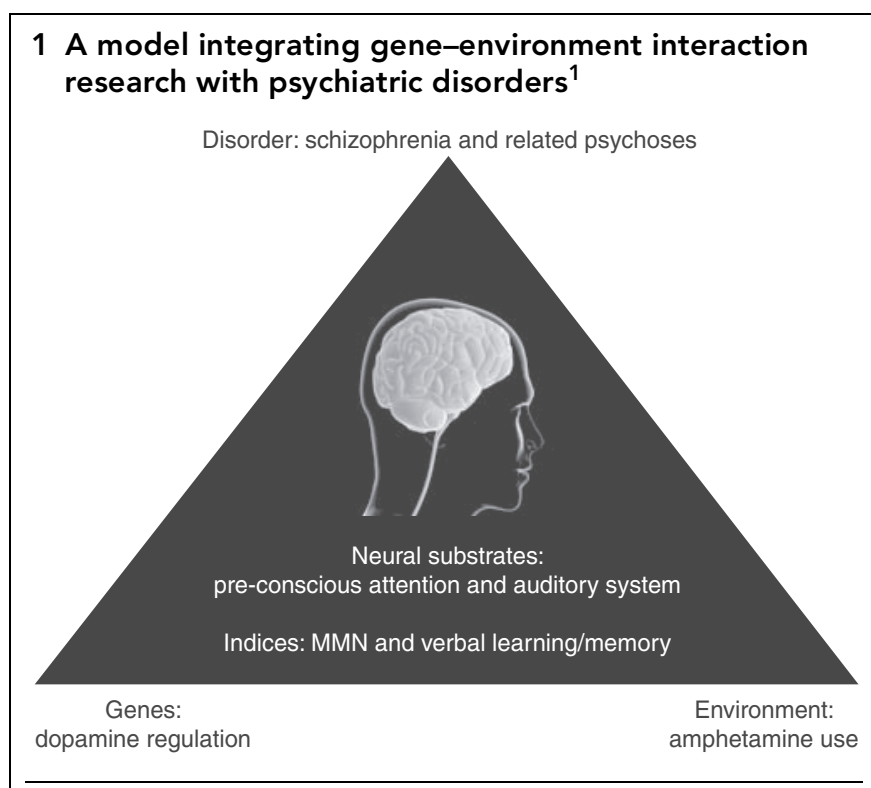

Here, amphetamine use and genotypic susceptibility are causally linked to psychosis. This model can be tested by neurobiological markers (eg, mismatch negativity [MMN] and verbal learning or memory), which index disrupted pre-conscious attention and a dysfunctional auditory system.

value. Compared with ATS users who do not develop psychoses, those experiencing psychosis report first using ATSs at a younger age, using greater amounts of ATSs, and have higher rates of premorbid schizotypal traits, major depression, alcohol dependence and antisocial personality disorder. ${ }^{5}$ These findings suggest similarities with young people who develop schizophrenia and related psychoses without ATS exposure.

\section{Genetic underpinnings of schizophrenia and related psychoses}

The consensus of family, twin and adoption studies is that there is a large genetic contribution to the vulnerability to schizophrenia and related psychoses. ${ }^{14}$ The high heritability demonstrated in such research led to a wave of linkage studies, and more recently genome-wide association studies, that have generally been inconclusive. ${ }^{2,14}$ Such limitations have precipitated a focus on biological indices, which lie somewhere between genes and behaviour. These endophenotypes are considered to be measurable components reflecting biological modulation. ${ }^{15}$ To be valid, endophenotypes must demonstrate an association with candidate genotypes (and/or genetic loci), heritability and functional links to symptoms. ${ }^{15}$ Candidate endophenotypes in schizophrenia include neurophysiological, neuroanatomical and neuropsychological variables.

As an exemplar candidate gene for schizophrenia and other psychoses, the gene encoding the enzyme catechol-O-methyltransferase (COMT) has clear functional links, due to its role in the catabolic clearance of dopamine (a neurotransmitter centrally involved in the expression of psychosis). ${ }^{16}$ The COMT gene is one of the most widely studied in psychiatric research, probably because of its function, location, and the apparent simplicity of its genotypic variation. ${ }^{16}$ Functional polymorphisms of COMT have been shown to be associated with neurophysiological and neuro- psychological indices. ${ }^{15}$ Despite this, it remains difficult to draw strong conclusions about COMT's role in schizophrenia, as studies have shown small effect sizes and poor reproducibility. ${ }^{16}$ However, the assessment of COMT in conjunction with the profound dopamine release associated with ATS use may shed light on the underlying neural substrate of psychosis.

Guided by its relevance to the dopamine system, val158met, a COMT functional polymorphism, has been implicated in ATS use, both with respect to its rewarding properties and the potential to develop psychosis. ${ }^{17}$ Furthermore, the interaction between COMT polymorphism, working memory performance and ATS response provides evidence that individual differences in baseline dopamine functioning plays a key role. ${ }^{18}$ One study found that ATS users who showed spontaneous psychosis relapse (without subsequent ATS use) were 1.7-fold more likely to have the COMT met allele (which increases dopaminergic signalling) compared with controls. ${ }^{19}$ That is, the presence of this allele appeared to increase the risk of an adverse (psychotic) response to ATS use. ${ }^{19}$ Another study of substance use reported that the val/val genotype of COMT in combination with adolescent cannabis use was strongly associated with the later development of psychosis. ${ }^{20}$ Overall, these findings suggest that the interaction between genes and environmental factors is complex. For example, persistent negative forms of psychosis may be associated with the met allele, whereas the val allele may be associated with episodic reactive forms of psychosis. ${ }^{21}$

\section{Candidate neurobiological markers of ATS-related psychosis}

\section{A neurophysiological marker}

The event-related potential (ERP) component, mismatch negativity (MMN), is an exemplar neurophysiological marker of schizophrenia. ${ }^{3,22}$ MMN reflects the neural detection of a mismatch between expected and deviant environmental inputs (stimuli). It indexes information processing at early (100-200 ms) stages, and is important for the preliminary organisation of subsequent responses to a significant or novel event. Notably, MMN precedes the conscious detection of change and is elicited in passive listening tasks (eg, the participant listens to a series of frequent standard tones that are randomly interrupted by infrequent deviant tones, which differ in pitch or duration). People with schizophrenia show a significantly reduced MMN response to deviant (typically auditory) tones, suggesting a dysfunctional detection system at early pre-attentive stages. MMN is associated with illness duration in schizophrenia (regardless of medication), and is thought to index neuropathological changes in the auditory cortex, which may contribute to the cognitive deficits seen in these individuals. 3,22

There is increasing research examining MMN in conjunction with cognitive measures. In particular, recent studies have shown that individuals with schizophrenia demonstrate decreased MMN and corresponding deficits in verbal learning and memory, ${ }^{22}$ working memory ${ }^{23}$ and cognitive flexibility. ${ }^{24}$ However, MMN deficits do not appear to occur in other major psychiatric disorders, including depression, bipolar disorder, obsessive-compulsive disorder and attention deficit hyperactivity disorder. ${ }^{3,22}$ To our knowledge, only one study has assessed MMN in ATS users; it did not address related psychosis. ${ }^{25}$ The authors reported a significant relationship between MMN reduction and duration of ATS use, 


\begin{tabular}{|c|c|c|}
\hline Clinical phenotype & Schizophrenia and related psychoses (without substance use) & ATS-related psychoses \\
\hline Age of onset & Typically 15 to 30 years & Typically 15 to 30 years \\
\hline At-risk personality traits & Schizotypy, ${ }^{*}$ schizoid, neuroticism & Schizotypy, schizoid \\
\hline Pre-psychotic phase & $\begin{array}{l}\text { Non-specific depressive features, social withdrawal. Changes in functioning, } \\
\text { thinking, behaviour and mood. Increased suspiciousness can occur }\end{array}$ & $\begin{array}{l}\text { To be determined. Some evidence of } \\
\text { increased suspiciousness }\end{array}$ \\
\hline Clinical presentation & $\begin{array}{l}\text { Includes: positive symptoms (eg, delusions, hallucinations, bizarre } \\
\text { behaviour); negative symptoms (eg, blunted affect, avolition, amotivation, } \\
\text { anhedonia, social withdrawal); cognitive symptoms (eg, difficulties in } \\
\text { attention, memory, problem solving) }\end{array}$ & $\begin{array}{l}\text { Typically transient psychosis with } \\
\text { hallucinations, suspiciousness and unusual } \\
\text { thought content. Disorganised speech } \\
\text { and negative symptoms may occur }\end{array}$ \\
\hline Longitudinal & Cognitive deterioration with relapse or recurrence of psychotic symptoms & $\begin{array}{l}\text { Cognitive problems with relapse or } \\
\text { recurrence of psychotic symptoms }\end{array}$ \\
\hline Neurophysiological & $\begin{array}{l}\text { Reduced auditory mismatch negativity. Reduced contextual updating } \\
\text { (indexed by reduced P300) }\end{array}$ & To be determined \\
\hline Neuropsychological & Poor verbal learning and memory, working memory, executive functioning & To be determined \\
\hline Neurobiological & Dysregulation of mesolimbic and cortical circuits & $\begin{array}{l}\text { Dysregulation of mesolimbic and cortical } \\
\text { circuits }\end{array}$ \\
\hline Neurochemical & $\begin{array}{l}\text { Positive symptoms reduced by dopamine } 2 \text { receptor blockade; } \\
\text { glutamatergic (NMDA receptor) dysfunction }\end{array}$ & $\begin{array}{l}\text { Positive symptoms exacerbated by } \\
\text { profound ATS-precipitated dopamine } \\
\text { release }\end{array}$ \\
\hline Susceptibility genotype & $\begin{array}{l}\text { Catechol-O-methyltransferase (COMT), dysbindin } 1 \text { (DTNBP1), neuregulin } \\
1 \text { (NRG1) }\end{array}$ & $\begin{array}{l}\text { COMT, protein interacting with } \mathrm{C} \text { kinase } \\
1 \text { (PICK1) }\end{array}$ \\
\hline Family history & Schizophrenia and related psychoses & Schizophrenia and related psychoses \\
\hline
\end{tabular}

ATS = amphetamine-type stimulant. * Schizotypy is a personality trait involving up to four factors: unusual experiences; cognitive disorganisation; introverted anhedonia and impulsive nonconformity.

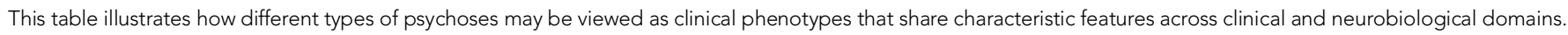

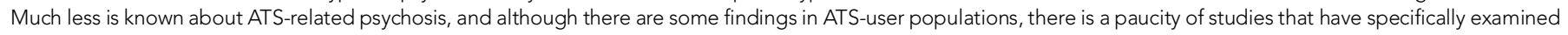
ATS-related psychosis itself. The ATS-related psychosis population can serve as an exemplar for the gene-environment-neuroscience interpretation of the psychoses.

suggesting that longer-term users have a greater impairment in pre-attentive information processing. ${ }^{25}$ Clearly, more research examining the MMN component in ATS users (and ATS-related psychosis) is required.

\section{Neuropsychological markers}

Cognitive deficits are at the core of dysfunction in schizophrenia, ${ }^{22,26}$ with verbal learning and memory, working memory and executive functions (eg, cognitive flexibility) being the most characteristically impaired. ${ }^{26-28}$ Verbal learning and memory and working memory deficits seen in schizophrenia may stem from a common dysfunction in encoding, ${ }^{27}$ or such deficits may reflect the inability to form and use transient memory traces. ${ }^{23}$ Whether these deficits reflect a generalised cognitive impairment or differential impairment across domains may be best determined by further examination of the relationships between mediotemporal (verbal learning and memory) and frontal (working memory and/or executive function) deficits seen in schizophrenia. This approach would be augmented by research examining ATS psychosis, particularly as there is clear overlap in the nature of cognitive deficits observed. Indeed, neuropsychological research with ATS users has found significant impairments in verbal learning and memory, executive functioning (eg, response inhibition and mental flexibility) and information processing speed, ${ }^{29}$ with evidence that such deficits are related to severity of ATS use, ${ }^{30}$ and may predict relapse following abstinence. $^{31}$

\section{Combinatorial evidence of ATS-related psychosis: early identification}

Our major assumption for working with the ATS-related psychosis model is that ATS-related psychosis and schizophrenia share common features and a common neural substrate (Box 2). That is, ATS-related psychosis relates to fundamental deficits in pre-attentional auditory processes and subsequent controlled processing, as indexed by neurophysiological (MMN) and neuropsychological (verbal learning and memory) markers, respectively. These markers are, in turn, mediated by gene-environment interactions, as particular genotypes (eg, COMT) differentially affect psychiatric outcomes associated with ATS use (ie, psychosis). Finally, identifying such markers in young people who use ATSs may assist with the early identification of individuals at risk of developing psychosis.

Future research on ATS-related psychosis must also consider the role of other neurotransmitters, such as glutamate. The hyper-dopaminergic state thought to be at the core of schizophrenia has been proposed to be a consequence of NMDA receptor hypofunction. ${ }^{32}$ Notably, healthy participants given the NMDA receptor antagonist ketamine, demonstrate reduced MMN, with concomitant increases in "psychotic" symptoms. ${ }^{33}$ Thus, the relationship between MMN and dopamine activity may be mediated by NMDA-glutamate function. Indeed, genetically elevated dopamine tone, coupled with heavy ATS use, may result in profound dopamine release, potentially resulting in glutamate-mediated neurotoxicity. If so, this may produce 
impairments in MMN and cognition that are common to both schizophrenia and ATS-related psychosis.

\section{Significance}

Psychotic disorders in young people are associated with high rates of disability and premature death. Both the clinical symptoms of psychosis and the associated cognitive impairment are indicative of significant neurobiological changes. Young people using ATSs are obviously at risk. Insufficient research has been invested in understanding the neurobiological link between ATS use and major psychiatric disorder. Studies are urgently needed to delineate the early neurobiological markers of subtle brain dysfunction (ie, the ERP marker of schizophrenia, MMN) in addition to other commonly used cognitive measures. These same neurobiological markers may be useful in those with psychoses without ATS exposure. In either group, such measures could be used to develop better early intervention and neuroprotective approaches. This is particularly relevant when onset of illness occurs during critical neurodevelopmental periods, such as adolescence and young adulthood, resulting in significant psychosocial impairment and poor developmental outcomes.

\section{Acknowledgements}

Daniel Hermens is supported by National Health and Medical Research Council (NHMRC) program grant (no. 350241) funding. Dan Lubman is supported by the Colonial Foundation. Sharon Naismith is supported by an NHMRC Clinical Research Fellowship.

\section{Competing interests}

None identified.

\section{Author details}

Daniel F Hermens, BSc, GDipSci, PhD, Research Fellow ${ }^{1}$

Dan I Lubman, PhD, FRANZCP, FAChAM, Associate Professor ${ }^{2}$

Philip B Ward, BMedSc, PhD, Associate Professor 3,4

Sharon L Naismith, BA, DPsych, CCN, Director, Clinical Research Unit ${ }^{1}$

Ian B Hickie, MD, FRANZCP, AM, Professor of Psychiatry and Executive Director ${ }^{1}$

1 Brain and Mind Research Institute, University of Sydney, Sydney, NSW. 2 ORYGEN Research Centre, University of Melbourne, Melbourne, VIC. 3 School of Psychiatry, University of New South Wales, Sydney, NSW. 4 Schizophrenia Research Unit, Liverpool Hospital, Sydney, NSW.

Correspondence: dhermens@med.usyd.edu.au

\section{References}

1 Caspi A, Moffitt TE. Gene-environment interactions in psychiatry: joining forces with neuroscience. Nat Rev Neurosci 2006; 7: 583-590.

2 Owen MJ, Williams NM, O'Donovan MC. The molecular genetics of schizophrenia: new findings promise new insights. Mol Psychiatry 2004; 9: 14-27.

3 Javitt DC, Spencer KM, Thaker GK, et al. Neurophysiological biomarkers for drug development in schizophrenia. Nat Rev Drug Discov 2008; 7: 6883.

4 Harris D, Batki SL. Stimulant psychosis: symptom profile and acute clinical course. Am J Addict 2000; 9: 28-37.

5 McKetin R, McLaren J, Lubman DI, Hides L. The prevalence of psychotic symptoms among methamphetamine users. Addiction 2006; 101: 1473 1478

6 Ross J, editor. Illicit drug use in Australia: epidemiology, use patterns and associated harm. 2nd ed. National Drug Strategy Monograph No. 63. Canberra: Commonwealth Department of Health and Family Services, 2007.
7 Chen CK, Lin SK, Sham PC, et al. Pre-morbid characteristics and comorbidity of methamphetamine users with and without psychosis. Psychol Med 2003; 33: 1407-1414.

8 Nordahl TE, Salo R, Leamon M. Neuropsychological effects of chronic methamphetamine use on neurotransmitters and cognition: a review. J Neuropsychiatry Clin Neurosci 2003; 15: 317-325.

9 American Psychiatric Association. DSM-IV sourcebook. Washington, DC: APA, 1994

10 Mathias S, Lubman D, Hides L. Substance-induced psychosis: a diagnostic conundrum. J Clin Psychiatry 2008; 69: 358-367.

11 Lambert M, Conus $\mathrm{P}$, Lubman DI, et al. The impact of substance use disorders on clinical outcome in 643 patients with first-episode psychosis. Acta Psychiatr Scand 2005; 112: 141-148.

12 Flaum M, Schultz SK. When does amphetamine-induced psychosis become schizophrenia? Am J Psychiatry 1996; 153: 812-815.

13 Zweben JE, Cohen JB, Christian D, et al. Psychiatric symptoms in methamphetamine users. Am J Addict 2004; 13: 181-190.

14 Tsuang MT, Stone WS, Faraone SV. Genes, environment and schizophrenia. Br J Psychiatry 2001; 178 Suppl: S18-S24.

15 Gottesman II, Gould TD. The endophenotype concept in psychiatry: etymology and strategic intentions. Am J Psychiatry 2003; 160: 636-645.

16 Williams HJ, Owen MJ, O'Donovan MC. Is COMT a susceptibility gene for schizophrenia? Schizophr Bull 2007; 33: 635-641.

17 Barr AM, Panenka WJ, MacEwan GW, et al. The need for speed: an update on methamphetamine addiction. J Psychiatry Neurosci 2006; 31: 301-313.

18 Mattay VS, Goldberg TE, Fera F, et al. Catechol O-methyltransferase val158-met genotype and individual variation in the brain response to amphetamine. Proc Natl Acad Sci USA 2003; 100: 6186-6191.

19 Suzuki A, Nakamura K, Sekine $Y$, et al. An association study between catechol-O-methyl transferase gene polymorphism and methamphetamine psychotic disorder. Psychiatr Genet 2006; 16: 133-138.

20 Caspi A, Moffitt TE, Cannon M, et al. Moderation of the effect of adolescent-onset cannabis use on adult psychosis by a functional polymorphism in the catechol-O-methyltransferase gene: longitudinal evidence of a gene $X$ environment interaction. Biol Psychiatry 2005; 57: 1117-1127.

21 Bilder RM, Volavka J, Lachman HM, et al. The catechol-O-methyltransferase polymorphism: relations to the tonic-phasic dopamine hypothesis and neuropsychiatric phenotypes. Neuropsychopharmacology 2004; 29: 1943-1961.

22 Umbricht D, Krljes S. Mismatch negativity in schizophrenia: a metaanalysis. Schizophr Res 2005; 76: 1-23.

23 Javitt DC, Shelley AM, Silipo G, et al. Deficits in auditory and visual context-dependent processing in schizophrenia: defining the pattern. Arch Gen Psychiatry 2000; 57: 1131-1137.

24 Oades RD, Wild-Wall N, Juran SA, et al. Auditory change detection in schizophrenia: sources of activity, related neuropsychological function and symptoms in patients with a first episode in adolescence, and patients 14 years after an adolescent illness-onset. BMC Psychiatry 2006; 6: 7.

25 Hosak L, Kremlacek J, Kuba M, et al. Mismatch negativity in methamphetamine dependence: a pilot study. Acta Neurobiol Exp (Wars) 2008; 68: 97102.

26 Elvevag B, Goldberg TE. Cognitive impairment in schizophrenia is the core of the disorder. Crit Rev Neurobiol 2000; 14: 1-21.

27 Danion J, Huron C, Vidailhet $P$, et al. Functional mechanisms of episodic memory impairment in schizophrenia. Can J Psychiatry 2007; 52: 693.

28 Kraus MS, Keefe RS. Cognition as an outcome measure in schizophrenia. Br J Psychiatry 2007; 50 Suppl: S46-S51.

29 Scott J, Woods S, Matt G, et al. Neurocognitive effects of methamphetamine: a critical review and meta-analysis. Neuropsychol Rev 2007; 17: 275297.

30 McKetin R, Mattick RP. Attention and memory in illicit amphetamine users: comparison with non-drug-using controls. Drug Alcohol Depend 1998; 50: 181-184.

31 Simon SL, Dacey J, Glynn S, et al. The effect of relapse on cognition in abstinent methamphetamine abusers. J Subst Abuse Treat 2004; 27: 59-66.

32 Lisman JE, Coyle JT, Green RW, et al. Circuit-based framework for understanding neurotransmitter and risk gene interactions in schizophrenia. Trends Neurosci 2008; 31: 234-242.

33 Umbricht D, Koller R, Vollenweider FX, et al. Mismatch negativity predicts psychotic experiences induced by NMDA receptor antagonist in healthy volunteers. Biol Psychiatry 2002; 51: 400-406.

(Received 10 Jul 2008, accepted 25 Sep 2008) 